\title{
Part-set cuing in option generation
}

\author{
Fabio Del Missier and Chiara Terpini \\ University of Trieste, Trieste, Italy
}

\begin{abstract}
Memory research on the part-set cuing effect has shown that providing some of the to-be-remembered items as cues is not always beneficial and, in some cases, may even hurt retrieval. However, part-set cuing has been sparsely investigated in option generation tasks. Thus, limited empirical evidence for the existence of the effect in option generation is available, and no convincing explanation has been provided yet. In order to fill these gaps, we carried out four experiments. In Experiment 1A, we observed a significant decrease in option generation performance when potential options were presented as cues. Experiment 1B showed that the effect can also be obtained in older adults. Experiments 2A and 2B provided evidence compatible with an inhibition-based explanation of the observed effects.
\end{abstract}

Imagine that you have received a $€ 30$ coupon that can be used to purchase a book. In order to select a book to buy, you decide to write a list of potential authors. Before compiling the list, you notice on the table a leaflet from a bookstore that you received via postal mail some days ago. You think that the leaflet may offer useful hints, so you take a look at it and read a number of authors' names. Then you compile the list of potential authors and make your final choice. This simple scenario raises important questions about option generation and the role of decision options as retrieval cues. Does suggesting options reduce option generation capacity? If providing suggestions hurts generation, why does this happen? The experiments presented in this article were designed to answer these questions.

Memory research has shown that cues sometimes backfire. This phenomenon, puzzling for theories of associative retrieval, is known as part-set cuing. Recently, some researchers have hypothesized that part-set cuing may have a significant role in explaining decision-making phenomena, such as superadditivity in probabilistic judgment (Sloman, Rottenstreich, Wisniewski, Hadjichristidis, \& Fox, 2004) and focusing in information acquisition (Del Missier, Ferrante, \& Costantini, 2007). According to these researchers, presented cues (options) might affect option generation, indirectly influencing judgment and choice. The consequences of part-set cuing in decision making could be far-reaching, because option generation is a fundamental subprocess in many real-life contexts (e.g., medical diagnosis, technical or mechanical troubleshooting, memory-based consumer choice).

Although memory-based theories of decision making (such as decision by sampling [Stewart, Chater, \& Brown, 2006] and HyGene [Thomas, Dougherty, Sprenger, \& Harbison, 2008]) assign a central role to memory-sampling and associative-cuing processes, very few experimental studies have empirically investigated the influence of part- set cuing on the generation of decision options. Thus, little evidence is available to support the presumed influence of part-set cuing on option generation. Moreover, no persuasive explanation for this effect in option generation has been empirically tested. In order to fill these gaps, we carried out four experiments. These will be presented after a brief review of research on part-set cuing in memory and option generation tasks.

\section{Part-Set Cuing in Memory Research}

Part-set cuing has been widely investigated in memory research. The majority of studies have used an episodic recall paradigm (e.g., Roediger, Stellon, \& Tulving, 1977; Slamecka, 1968, 1969). In a typical experiment, two groups of participants study a list of words prior to a free recall task. Upon retrieval, some of the studied words are provided as cues to the participants in the experimental group (but not to the participants in the control group). Performance is evaluated by comparing the number of noncue words retrieved by the two groups.

The results obtained in these studies have depended on the number and type of cues presented. When participants study a series of words drawn from specific categories and a single cue for each category is presented, recall is usually facilitated (e.g., Hudson \& Austin, 1970). However, when a number of cues for each category are presented (or only a subset of target categories are cued), cues usually do not facilitate performance. The same happens when each item of the study list belongs to a different category. In these cases, participants in the experimental group usually retrieve fewer noncue words than do control participants, or no difference is found between the groups (see Nickerson, 1984; Roediger \& Neely, 1982).

A different paradigm for studying the part-set cuing effect is based on the generation of items from semantic categories (Brown, 1968; Karchmer \& Winograd, 1971). In 
these experiments, participants in the experimental group are required to study a list of items belonging to a given category (e.g., states of the U.S.). After the study period, these participants are asked to generate the maximum possible number of items belonging to the same category. The control group carries out the same generation task without having studied the cues (e.g., the states) beforehand. An alternative procedure involves presenting the cues just before the generation task (e.g., Sloman, 1991). A common result is that participants exposed to the cues produce fewer noncue responses than do participants who did not study (or read) them.

A series of explanations for the part-set cuing effect has been advanced over the years (see Nickerson, 1984). Some of these explanations have been dismissed on empirical grounds (e.g., editing, list-length hypothesis). Currently, the two general explanations that appear to be supported more by existing evidence are retrieval inhibition and strategy disruption (see Bäuml \& Aslan, 2006). According to the retrieval inhibition view (Anderson, E. L. Bjork, \& R. A. Bjork, 1994; Bäuml \& Aslan, 2004), the presentation of cues inhibits other items belonging to the target set. In particular, Bäuml and Aslan (2006) suggested that "the presentation of part-list cues leads to covert retrieval of the cue items at test ... covert retrieval is assumed to cause inhibition of the noncue items" (p. 34). The inhibition view has been empirically supported in studies on episodic recall (e.g., Bäuml \& Aslan, 2004, 2006). Given that the effects of inhibition take some time to dissipate, one of the most convincing pieces of evidence for the inhibition explanation is represented by the fact that part-set cuing can be lasting (i.e., it persists in a delayed test when the cues are not presented).

An alternative explanation of part-set cuing assumes that the presentation of cues disrupts a natural and effective retrieval strategy that participants would have used if the cues had not been presented (B. H. Basden, D. R. Basden, \& Stephens, 2002; D. R. Basden \& B. H. Basden, 1995). This explanation has received support from studies that varied the compatibility between the way cues were presented and the "natural" retrieval strategy triggered by the task. Moreover, some studies have shown that the removal of cues can produce a release from part-set cuing (e.g., D. R. Basden \& B. H. Basden, 1995; D. R. Basden, B. H. Basden, \& Galloway, 1977). However, there is also evidence against the strategy disruption explanation (e.g., Serra \& Oswald, 2006), and this account seems unable to explain some findings. In particular, it has been shown that part-set cuing effects can be obtained also when itemspecific cues have been employed, as in recognition (e.g., Oswald, Serra, \& Krishna, 2006) and word completion (e.g., Bäuml \& Aslan, 2004).

Recently, Bäuml and Aslan (2006) suggested that retrieval inhibition and strategy disruption can be reconciled. In particular, strategy disruption could explain partset cuing if an efficient retrieval plan based on interitem associations (chainlike associations created at encoding) were used by participants (given that they had not seen the cues), whereas inhibition would apply if this plan were not available.

\section{Part-Set Cuing in Option Generation}

Very few studies have investigated part-set cuing outside the memory arena. In a series of experiments, Peynirciouğlu (1987) found the effect in four nonmemory tasks involving verbal problem solving, visual attention, recognition, and imagination. She concluded that the effect of part-set cuing "encompasses more than just memory. It is a more general phenomenon in which partial answers or hints may sometimes serve to block the general thinking or cognitive process instead of, as intuition would suggest (at least in the realm of memory), facilitate it" (p. 440). To the best of our knowledge, only four studies have investigated the part-set cuing effect in option generation.

Alba and Chattopadhyay (1985) examined the generation and retrieval of brand names of given product classes in a sample of consumers. In a first experiment, the number of brand names generated by participants decreased with the increase in the number of presented cues. A second experiment showed that presenting cues produced a part-set cuing effect in the retrieval of brand names in men but not in women (allegedly more expert in the investigated domain). In a third experiment, the presentation of two cues for a given subcategory reduced the number of brand names generated from uncued response subcategories, even if the participants were explicitly invited to generate options from subcategories other than the cued one.

Sloman (1991, Experiment 2) found a part-set cuing effect in a task requiring undergraduates to generate reasons in support of or against a given possibility (Bush administration would continue the Star Wars program; I would go to graduate school). Providing one (Star War scenario) or two (graduate school scenario) potential reasons as cues hindered the generation of other reasons. However, the presentation of cues did not affect subsequent likelihood judgments concerning each issue.

Pei and Tuttle (1999) studied the influence of the presentation of cues (potential diagnoses) on the recall and generation of options in a financial domain (diagnosis of accounting anomalies). They observed the effect only for some conditions in three experiments (when lowplausibility diagnoses were presented as cues) and explained it by referring to the editing account (Roediger \& Tulving, 1974). ${ }^{1}$ However, the editing explanation seems to be a rather implausible explanation for their generation experiment, given that a very small number of cues were presented and there was apparently no strict time limit for the accomplishment of the task. Furthermore, the editing explanation does not seem to be a valid account in general, because it is unable to explain the effects obtained in various kinds of part-set cuing studies (see Nickerson, 1984).

Tomlinson (2007) analyzed the relationship between the part-set cuing effect and probabilistic judgment. To assess the potential role of part-set cuing as a cause of the implicit superadditivity effect, Tomlinson replicated Sloman et al.'s (2004) study, adding a generation task just after the probabilistic judgment (Experiment 1). This task was used to appraise the number of options generated for the judgment as a function of presented cues (unpacked/packed options in the description of the judgment problem). Al- 
though replicating Sloman et al.'s results for probabilistic judgment, Tomlinson did not observe part-set cuing effects (see also her Experiment 3).

To summarize, part-set cuing in the generation of decision options was observed in some experiments, but not always. Furthermore, no convincing explanation of the effect has been tested in option generation domains.

\section{Motivation and Plan of the Experiments}

The studies we have reviewed in the previous sections have pointed out two important research issues related to part-set cuing. First, we still do not know to what extent the part-set cuing effect can be reliably observed in tasks other than retrieval of word lists or the generation of items from well-defined semantic categories. In particular, it is not clear whether the part-set cuing effect can be reliably observed in option generation. Second, a convincing explanation for part-set cuing effects in generation tasks (including option generation) is still needed. Even if plausible explanations have been proposed and tested in the episodic domain, they have remained experimentally untested in generation paradigms.

In order to fill these gaps, we carried out four experiments. First, we investigated the part-set cuing effect in various decision-making scenarios, both in undergraduates (Experiment 1A) and in wealthy older participants attending U3A courses (Experiment 1B). These experiments were aimed at providing clear empirical evidence for the existence of the effect in option generation and to show that the effect is robust to contentual, procedural, and sample variations. The main goal of the following two experiments (2A and $2 \mathrm{~B}$ ) was, instead, to identify an explanation of the part-set cuing effect in option generation. In these experiments, we contrasted the two accounts that are actually supported more by memory research (inhibition and strategy disruption) and that have also been considered as potential explanations of part-set cuing effects in more complex tasks (e.g., Peynirciouğlu, 1987; Roediger \& Neely, 1982). To this end, Experiments 2A and 2B employed the test-retest procedure (see Bäuml \& Aslan, 2006) within a generation paradigm, applying it to the decision scenarios in which we observed the strongest part-set cuing effects.

\section{EXPERIMENT 1A}

Experiment 1A was planned with the aim of providing evidence for the part-set cuing effect in option generation in a sample of young adults. Adopting the generation paradigm, we investigated whether part-set cuing effects could be observed when participants were required to produce options in response to different decision scenarios. At the same time, in order to further assess the robustness of our findings, we manipulated the number of cues presented to the participants.

\section{Method}

Participants, Design, and Hypothesis

One hundred eighty-four undergraduates from the Universities of Trieste and Padua (60 of them male and 124 female; age, $M=$
22 years, $S D=4)$ took part in the experiment. Data were collected in class meetings. We adopted a 3 (number of cues) $\times 3$ (scenario) mixed design. The number of cues was varied between subjects, and the type of scenario (radio, gift, or author) was a within-subjects variable. The participants were randomly assigned to three groups (weak cuing, $n=78$; strong cuing, $n=76$; control [no cuing], $n=30$ ). ${ }^{2}$

According to the part-set cuing hypothesis, participants in the control condition should generate a higher number of noncue options than participants in the experimental conditions (weak cuing and strong cuing). This hypothesis should hold true regardless of the type of scenario and the number of cues.

\section{Procedure}

The participants received a booklet containing written instructions, descriptions of the scenarios (see Table 1), and response pages. Following the instructions, they went through the three scenarios, which were presented sequentially. For each scenario, they were asked to generate the maximum number of possible options in 5 min. ${ }^{3}$ Experimenters timed the participants' performance. Sociodemographic information was collected on the last page of the booklet.

The participants in the control group were requested to read the scenario and write down the maximum number of possible options within the time limit. The participants in the experimental groups were given a number of potential options as cues for the generation task. These cues were made available both before and during the generation task through a sequence of three steps. First, the participants read the scenario. Then, on the same page of the booklet, the participants were asked to evaluate each cue for its likelihood as cause (in the radio scenario) or for the probability of being purchased by them (in the gift and author scenarios) on a 7-point rating scale. ${ }^{4}$ The participants were allowed $3 \mathrm{~min}$ for completing this rating task. Finally, on the next page of the booklet, the scenario was presented again (followed by the list of cues), and the participants were asked to write the maximum number of possible options they could.

\section{Materials}

Scenarios. We selected three problems representative of different decision situations (see Table 1), which could be used with both young and older adults. These scenarios were selected for their face validity and after an in-depth analysis of the materials used in previous option generation studies. Each problem refers to a simple but nonroutine situation in which options have to be generated from an ad hoc category (Barsalou, 1983). We avoided routine or very familiar situations because, in these cases, the decision maker usually does not generate several options, focusing instead on the few alternatives that are more available (e.g., Johnson \& Raab, 2003).

Table 1

Decision Scenarios Used in the Experiments

\begin{tabular}{|c|c|c|}
\hline Scenario & Experiment & Text \\
\hline Radio & $1 \mathrm{~A}, 1 \mathrm{~B}, 2 \mathrm{~A}$ & $\begin{array}{l}\text { Imagine you are home. You hear the sound } \\
\text { of the radio coming from the living room. } \\
\text { They are broadcasting the news. Suddenly, } \\
\text { you don't hear the speaker's voice. What } \\
\text { could have happened? It could have hap- } \\
\text { pened that ... }\end{array}$ \\
\hline Gift & $1 \mathrm{~A}, 1 \mathrm{~B}$ & $\begin{array}{l}\text { Imagine having received } € 30 \text { that you can } \\
\text { use to buy a gift for yourself. You have to } \\
\text { decide what to buy. A gift you could buy } \\
\text { for yourself with } € 30 \text { is ... }\end{array}$ \\
\hline Author & $1 \mathrm{~A}, 1 \mathrm{~B}$ & $\begin{array}{l}\text { Now imagine having decided to buy a } \\
\text { book with the } € 30 \text { you received. An author } \\
\text { of books you could buy is... }\end{array}$ \\
\hline Author & $2 \mathrm{~B}$ & $\begin{array}{l}\text { Imagine having received } € 30 \text { that you can } \\
\text { use to buy a book. An author of books you } \\
\text { could buy is... }\end{array}$ \\
\hline
\end{tabular}


The radio scenario is a diagnostic task. We selected it because option generation is common in diagnosis, and diagnosis is an important decision-making activity. Previous studies on option generation have used diagnostic scenarios. Mehle (1982) asked experts and novices in automobile troubleshooting to generate possible reasons that would explain a series of automobile problems. Pei and Tuttle (1999) required professional auditors to list possible causes of accounting errors. However, the tasks used in previous studies require some degree of domain-specific knowledge. In the radio scenario, options can be easily generated even if participants have no domainspecific knowledge.

The gift scenario represents another common decision-making situation: option generation in view of a purchase (see Alba \& Chattopadhyay, 1985). A specific constraint on the gift price was placed, in order to limit the participants' potential reliance on stereotyped responses. Finally, the author scenario represents another purchaserelated situation. In this case, however, the task is closer to the semantically rich movie stars generation employed by Foos and Clark (2000).

Cue selection. We selected the cues for the radio and gift scenarios from a pool of options generated in a pilot study by a group of 40 undergraduates at the University of Trento (31 of them female and 9 male; age, $M=23.6$ years, $S D=6.19$ ). In this study, akin to the control conditions in Experiments $1 \mathrm{~A}$ and 1B, the participants were asked to generate the maximum possible number of options in response to each scenario. Then, the participants' answers were classified by two independent judges (with interrater agreement always greater than $90 \%$ and resolution of disagreements by joint discussion), and the frequency of production of each response was computed. Finally, cues were selected from the pool of classified options according to the following four criteria (categories and cues are presented in the Appendix).

1. Part-category cuing. For each scenario, we did not select cues from every potential response category. More than one cue for each of the cued categories was presented, but some categories were not cued (see Nickerson, 1984). This type of cuing avoided depleting the number of potential responses, allowing the participants to generate options from either cued or uncued categories. Moreover, this kind of cuing is likely to occur in real decision-making contexts.

2. Frequency of generation. In agreement with previous studies, options with a medium-to-low frequency of generation were selected as cues for the radio and the gift scenarios (i.e., items produced by no more than $35 \%$ of the participants in the pilot test). Given that items with a higher frequency of generation are usually more affected by part-set cuing (e.g., Kissler \& Bäuml, 2005), using low-frequency exemplars as cues should increase the power of the test. However, for the author scenario, we selected well-known names of writers, in order to avoid the risk that the participants might fail to recognize them (thus making cuing nonsensical). To this aim, we prepared a list of allegedly known authors that could be classified into four categories (Italian authors of novels, non-Italian authors of novels, authors of crime fiction, authors of political/historical essays). Then we asked 20 participants ( 10 young and 10 older adults, balanced for gender) to rate each author for its popularity on a 5-point scale ranging from unknown to the general public to well-known to the general public. Finally, we selected the cues from the authors who received the highest average evaluations.

3. Cue validity across age and gender. We selected items that may represent plausible options for both young and older adults. At the same time, we selected items that appeared to be suited for both males and females.

4. Number of cues. In the strong-cuing condition, we used 24 options for the gift and the author scenarios. This figure is in line with the number of cues presented in previous memory studies carried out with the generation paradigm (see Brown, 1968; Foos \& Clark, 2000 ), and it represents a relatively small proportion of the potential number of responses, as estimated by the pooled group performance in the pilot study ( $26 \%$ in the gift scenario). In the weak-cuing conditions for the gift and author scenarios, the number of cues was re- duced by one fourth (i.e., 18 cues were used). For the radio scenario, we decided to present a smaller absolute number of cues, because pilot testing had shown that participants were able to generate fewer options in response to this problem. Therefore, we presented 16 cues in the strong-cuing condition and 12 cues in the weak-cuing condition (again, with a reduction of one fourth in the number of cues across conditions). These figures corresponded to $44 \%$ and $33 \%$ of the total items produced in the pilot test (percentages lower than the $50 \%$ cues-to-responses ratio adopted in previous memory studies: e.g., Brown, 1968; Foos \& Clark, 2000).

\section{Results}

Following a procedure common in experiments on partset cuing, in all the experiments described in this article, we compared the number of noncue options produced in the experimental condition with the number of noncue options produced in the control condition. This implied creating a different control condition for each experimental condition, editing out the cues used in each experimental condition from the participants' responses in the control group. Following this procedure, we created a control condition for each of the experimental groups, and we analyzed the results with a $2 \times 3$ mixed ANOVA for each level of cuing. ${ }^{5}$

The participants in the weak-cuing condition generated fewer noncue options than did the participants in the control condition (Figure 1, left panel), as shown by the main effect of cuing $\left[F(1,105)=13.38, M S_{\mathrm{e}}=17.37, p<.001\right.$, $\left.\eta^{2}=.11\right]$. The main effect of scenario was also significant $\left[F(2,210)=44.47, M S_{\mathrm{e}}=8.76, p<.0001, \eta^{2}=.30\right]$, as well as the cuing $\times$ scenario interaction $[F(2,210)=8.84$, $\left.M S_{\mathrm{e}}=8.76, p<.001, \eta^{2}=.07\right]$. Planned comparisons showed that the part-set cuing effect was significant in the radio scenario ( $p<.001$; weak cuing, $M=3.87$; control, $M=5.41)$ and in the author scenario $(p<.0001$; weak cuing, $M=5.01$; control, $M=9.00)$, but not in the gift scenario ( $p=.80$; weak cuing, $M=8.82$; control, $M=$ 9.03).

The participants in the strong-cuing condition generated fewer noncue options than did the participants in the control condition $\left[F(1,102)=18.55, M S_{\mathrm{e}}=14.53, p<\right.$ $.0001, \eta^{2}=.15$; Figure 1, right panel]. The main effect of scenario was significant $\left[F(2,204)=35.70, M S_{\mathrm{e}}=9.81\right.$, $\left.p<.0001, \eta^{2}=.26\right]$, but not the interaction $[F(2,204)=$ $\left.1.86, M S_{\mathrm{e}}=9.81, p=.16\right]$. Planned comparisons showed significant part-set cuing effects in the radio scenario ( $p<.0001$; strong cuing, $M=3.06$; control, $M=4.83$ ) and in the author scenario ( $p<.01$; strong cuing, $M=$ 5.60; control, $M=8.72$ ), whereas the difference in the gift scenario was marginally significant $(p=.07$; strong cuing, $M=7.08$; control, $M=8.41$ ).

\section{Discussion}

The part-set cuing hypothesis was clearly supported in two of the decision domains we investigated (radio and author). In the third domain (gift), cues never facilitated option generation, and we detected a marginally significant difference in the expected direction when a higher number of cues were presented. The global pattern of the results supports the hypothesis that providing cues re- 

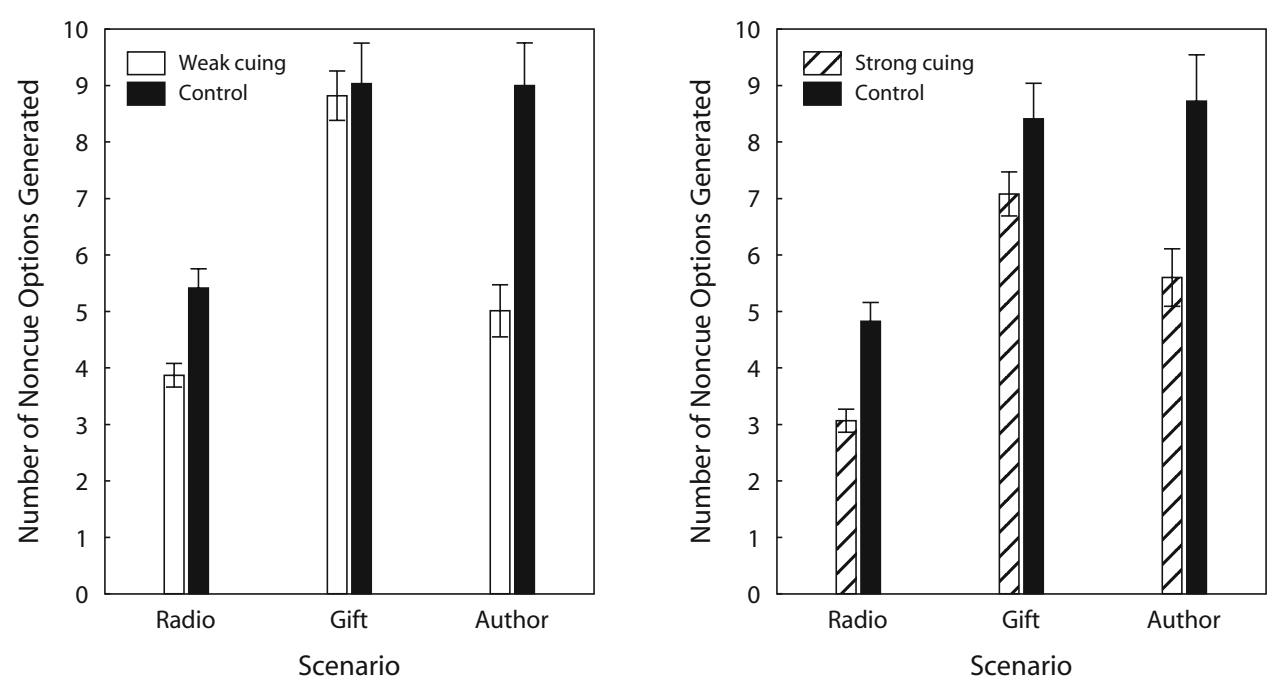

Figure 1. Number of noncue options generated in Experiment 1A $( \pm S E)$ as a function of experimental condition and decision scenario (radio, gift, or author). The graph on the left panel shows the results for the weak-cuing condition and its associated control condition. The graph on the right panel shows the results for the strong-cuing condition and its associated control condition.

duces option generation capacity. However, differences between scenarios show that the strength of the effect may depend, to some extent, on the decision problem. It is possible that the ad hoc category used for gift generation was better established in the participants' memory than the categories related to the other scenarios or that stronger preexperimental interitem associations existed. According to Bäuml and Aslan (2006), no part-set cuing should occur when items are strongly associated and integrated in memory (see also Bäuml \& Kuhbandner, 2003).

\section{EXPERIMENT 1B}

Experiment 1B was carried out with the aim of investigating part-set cuing in the generation of decision options in a sample of older adults. The main goal of this experiment was to generalize the results of Experiment 1A to a different population. Additional aims of the experiment were to contribute to the debate on part-set cuing in aging by providing novel evidence from generation tasks and to examine option generation performance in older participants.

In recent years, part-set cuing has been sparsely investigated in older participants, but different studies have obtained contrasting results. Marsh, Dolan, Balota, and Roediger (2004) found part-set cuing effects in three experiments carried out with the episodic recall paradigm (as did Moulin, 2000). Using the generation paradigm, Foos and Clark (2000) found, instead, that older participants benefited from cues in a specific domain (generation of movie stars' names). They explained this result by assuming that, due to the reduced efficiency of inhibitory processes, older participants are less affected by cue-induced retrieval blocks.

According to our opinion, a single counterintuitive result in a specific generation domain is not sufficient to conclude that cue facilitation will be usually observed in generation tasks performed by older adults, given that part-set cuing has been consistently observed in a series of experiments on episodic recall. Therefore, part-set cuing effects (or no facilitation) should be observed also in generation tasks when the participants' inhibitory capacity is not markedly deteriorated (as in normal aging).

\section{Method}

\section{Participants}

Older participants attending U3A courses in Trento agreed to participate in our experiment $(n=72,16$ of them male and 56 female; age, $M=69$ years, $S D=7$ ). They were generally well educated (38\% reported having a high school or college degree, $37 \%$ a middle school degree, and $24 \%$ an elementary school degree, and $1 \%$ did not specify), and they reported being in good health.

\section{Design, Hypothesis, Procedure, and Materials}

We followed the 3 (number of cues) $\times 3$ (scenario) mixed design already adopted in Experiment 1A. The number of cues was varied between subjects, and the type of scenario was manipulated within subjects. The participants were randomly and evenly assigned to three groups (weak cuing, strong cuing, and control). We tested again the part-set cuing hypothesis, expecting to observe a higher number of noncue options generated in the control group (no cuing) than in the experimental groups. The procedure and materials were the same as those in Experiment 1A.

\section{Results}

As in the previous experiment, two control conditions, corresponding to the weak- and strong-cuing groups, were obtained from the control group. We analyzed the results with a $2 \times 3$ mixed ANOVA for each level of cuing (weak cuing or strong cuing). The dependent variable was the number of noncue options.

The $2 \times 3$ mixed ANOVA relative to the weak-cuing condition (Figure 2, left panel) showed again a main ef- 

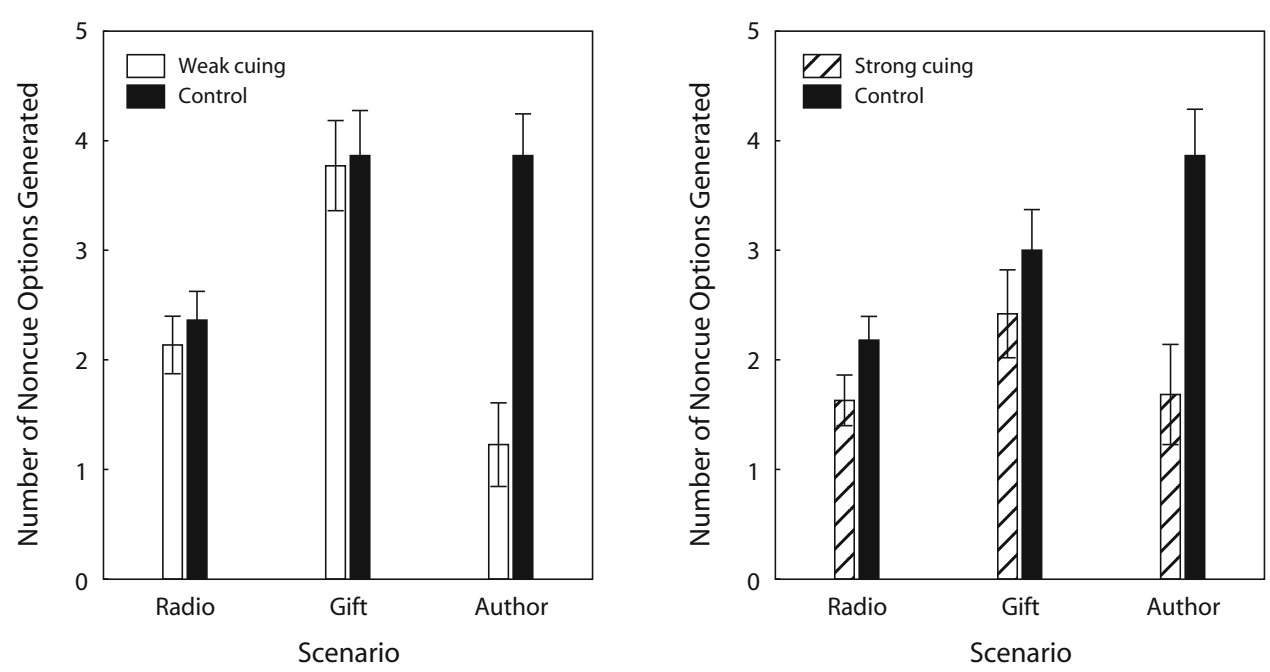

\begin{abstract}
Figure 2. Number of noncue options generated in Experiment 1B $( \pm S E)$ as a function of experimental condition and decision scenario (radio, gift, or author). The graph on the left panel shows the results of the weak-cuing condition and its associated control condition. The graph on the right panel shows the results of the strong-cuing condition and its associated control condition.
\end{abstract}

fect of cues $\left[F(1,42)=7.52, M S_{\mathrm{e}}=4.26, p<.01, \eta^{2}=\right.$ $.15]$ and of scenario $\left[F(2,84)=14.60, M S_{\mathrm{e}}=2.09, p<\right.$ $\left..0001, \eta^{2}=.25\right]$ and also highlighted a significant interaction $\left[F(2,84)=10.78, M S_{\mathrm{e}}=2.09, p<.0001, \eta^{2}=\right.$ .20]. Planned comparisons indicated a significant partset cuing effect in the author scenario $(p<.0001$; weak cuing, $M=1.23$; control, $M=3.86$ ), but not in the radio scenario ( $p=.57$; weak cuing, $M=2.14$; control, $M=$ 2.36 ) or in the gift scenario ( $p=.88$; weak cuing, $M=$ 3.77; control, $M=3.86$ ).

In the strong-cuing condition (Figure 2, right panel), the participants in the control condition generated more noncue options than did the participants in the experimental group $\left[F(1,39)=7.96, M S_{\mathrm{e}}=4.67, p<.01, \eta^{2}=.17\right]$. The main effect of scenario $\left[F(2,78)=5.68, M S_{\mathrm{e}}=1.68\right.$, $\left.p<.01, \eta^{2}=.13\right]$ and the interaction $[F(2,78)=5.28$, $\left.M S_{\mathrm{e}}=1.68, p<.01, \eta^{2}=.12\right]$ were also significant. Planned comparisons showed a significant difference in the author domain ( $p<.01$; strong cuing, $M=1.68$; control, $M=3.86$ ) and a marginally significant difference in the radio domain ( $p=.09$; strong cuing, $M=1.63$; control, $M=2.18$ ), whereas the difference in the gift scenario was not significant ( $p=.29$; strong cuing, $M=2.42$; control, $M=3.00$ ).

Finally, we compared option generation performance in young and older adults by contrasting the outcomes of Experiments 1A and 1B. A 3 (number of cues) $\times 3$ (scenario) $\times 2$ (age group) ANOVA showed that the older participants generated fewer options than did the young participants [main effect of age, $F(1,241)=138.72$, $\left.M S_{\mathrm{e}}=15.22, p<.0001, \eta^{2}=.36\right]$. Tukey HSD tests highlighted significant differences between the young and the older participants for the gift and the author scenarios ( $p<.0001$ for each test) and a marginally significant difference for the radio scenario $(p=.08)$. However, these results should be considered with caution, because they are based on a cross-experiment comparison involving groups of participants that might differ for various reasons.

\section{Discussion}

Experiment 1B showed significant part-set cuing effects in older adults, but it seems that the presence of the effect depended on the decision problem. However, as in Experiment 1A, the presentation of cues never facilitated option generation. The results obtained in the author scenario (and, to some extent, in the radio scenario) show, instead, that older adults can be prone to part-set cuing effects also in generation tasks, and not only in episodic retrieval. Finally, the cross-experimental comparison between young and older adults suggests that option generation performance significantly declines with age. In Experiment $1 \mathrm{~B}$, older adults' poorer generation performance (together with the relatively small number of participants) may have hindered the detection of part-set cuing effects. ${ }^{6}$ In any case, the results partially generalize the findings of Experiment $1 \mathrm{~A}$, by showing that part-set cuing effects can be observed also in older adults performing a generation task.

Future research should reconcile our findings (and the results obtained by Marsh et al., 2004, and Moulin, 2000) with the results of the study carried out by Foos and Clark (2000). This integration might be accomplished by assuming that part-set cuing effects are usually observed in older adults, both in generation tasks and in episodic retrieval, and that a boundary condition for the effect in generation tasks is represented by the efficiency of inhibitory processes. Thus, part-set facilitation might be observed only when the efficiency of inhibitory processes is significantly decreased. In order to test this possibility, future 
studies might include measures of individual differences in inhibition as control variables.

\section{EXPERIMENT 2A}

Experiments 2A and 2B were planned so as to explain the part-set cuing effect in option generation observed in our previous experiments. To this aim, we contrasted the two accounts that are actually supported more by memory research (inhibition and strategy disruption), which have been suggested also as potential explanations of part-set cuing effects in more complex generation tasks. This contrast was carried out through the application of the testretest procedure to each decision scenario in which we had previously observed a clear part-set cuing effect (radio in Experiment 2A and author in Experiment 2B). The episodic form of the test-retest procedure (e.g., Bäuml \& Aslan, 2006) requires that, after the study phase, participants perform two retrieval tests, separated by a filler task. Cues are presented to participants in the experimental group only in the first test, but they are not available in the delayed test. Participants in the control group perform the task without seeing the cues.

If part-set cuing is caused by cue-driven inhibition of potential responses, it should be observed also in the delayed test, due to the persistence in time of inhibitory effects (e.g., Anderson et al., 1994; Anderson \& Bjork, 1994). According to this account, the inhibition of target items consists, indeed, in a long-term change in their activation levels: "The detrimental effect of cues should reflect long-term changes in the items' activation levels and thus be lasting" (Bäuml \& Aslan, 2006, p. 34). Partset cuing effects have been actually observed in repeated tests after intervals of 2 min (Bäuml \& Aslan, 2006) and 10-12 min (Aslan, Bäuml, \& Grundgeiger, 2007). Assuming that the inhibitory effect of cuing remains stable across the two tests (e.g., Bäuml \& Aslan, 2006), only a main effect of cuing will be observed, and no release from part-set cuing will appear in the second test (i.e., no condition $\times$ test interaction will be detected).

However, if part-set cuing is due to the disruption of a natural and effective retrieval strategy (altered retrieval in the presence of cues), the effect should, instead, disappear in the delayed test where no cues are presented, because the participants in the experimental group will be able to turn back to their effective strategy soon after the removal of cues (D. R. Basden \& B. H. Basden, 1995). Thus, in the repeated test, they will attain the same level of performance as the participants in the control group. In other words, "the removal of cues should enable participants to turn back to their original strategies and thus eliminate any forgetting the cues originally produced" (Bäuml \& Aslan, 2006, p. 34). This release from part-set cuing in the repeated test will lead to a peculiar condition $\times$ test interaction.

As was reported in our brief introductory review of part-set cuing research, there is evidence supporting the view that inhibition and strategy disruption can explain different kinds of part-set cuing effects in the episodic par- adigm. Moreover, both of these explanations can be generalized to explain part-set cuing in generation performance (e.g., Peynirciouğlu, 1987; Roediger \& Neely, 1982).

In Experiments 2A and 2B, we employed the test-retest procedure within the generation paradigm, requiring the participants to perform two generation trials separated by a nonverbal filler task. As in the episodic paradigm, the cues were presented only in the first trial in the experimental conditions, and they were always removed in the second trial. In Experiment 2A, we also carried out an in-depth analysis of experimental results, in order to understand whether the part-set cuing effect we observed originates from an intercategorical effect of cues (partcategory cuing), from an intracategorical effect of cues ("traditional" part-set cuing), or from both these effects. Finally, we appraised the robustness of part-set cuing to variations in the cuing procedure. In particular, the participants in two different experimental groups were asked either to rate the cues (as in Experiments 1A and 1B) or simply to read the cues attentively.

\section{Method}

\section{Participants, Design, and Hypotheses}

One hundred twenty-one undergraduates recruited at the University of Trieste participated in our study (86 of them male and 35 female; age, $M=23$ years, $S D=3$ ). Data were collected in class meetings. Experiment $2 \mathrm{~A}$ followed a 3 (type of cuing) $\times 2$ (test) mixed design. Type of cuing (reading, $n=40$; rating, $n=40$; control-no cues, $n=41$ ) was varied between subjects, and test was a within-subjects variable. Only the radio scenario was employed in Experiment 2A. No difference in the part-set cuing effect was expected as a consequence of the variation of the cuing procedure (i.e., reading cues vs. rating cues).

According to the part-set cuing hypothesis, in the first test, we should observe a higher number of noncue options generated in the control condition than in each of the cuing conditions. If the inhibition hypothesis holds, we should find the same difference also in the delayed test, after the removal of the cues (i.e., main effect of cuing, but no significant type of cuing $\times$ test interaction). According to the strategy disruption hypothesis, we should instead observe a release from part-set cuing in the delayed test (i.e., a significant cuing $\times$ test interaction). Given that the radio scenario described a rather infrequent situation involving an ad hoc category (possible causes of the radio problem), we expected that the inhibition hypothesis was more likely to be supported. In fact, individuals may not hold preferred and effective strategies for generating items from ill-established ad hoc categories (see Barsalou, 1983) and, without a preferred generation strategy to be disrupted, strategy disruption should not occur.

\section{Procedure and Materials}

The participants in the rating group were cued with the procedure already adopted in Experiments 1A and 1B. After reading the scenario, they first rated the cues (in $3 \mathrm{~min}$ ) and then generated the maximum possible number of options in the presence of cues (in $5 \mathrm{~min}$ ). The participants in the reading group were asked to read the scenario, then to read the cues attentively (in $3 \mathrm{~min}$ ), and finally they had to generate the maximum possible number of options in the presence of cues (in $5 \mathrm{~min}$ ). The participants in the control group were first asked to perform a visuospatial filler task for $3 \mathrm{~min}$ (a find-the-difference puzzle), and then, after reading the scenario, they were required to generate the maximum possible number of options (in $5 \mathrm{~min}$ ). No cues were presented in the control condition.

After the first generation test, the participants in each group followed the same procedure. First, they performed a visuospatial filler 
task for $3 \mathrm{~min}$ (another find-the-difference puzzle). Then they were asked to perform the generation task a second time (with no cues available). Each task was presented in a different page of a booklet and was timed by the experimenter.

\section{Results}

\section{Generated Options}

The results were analyzed through a 3 (type of cuing) $\times$ 2 (test) ANOVA on the number of noncue options generated. ${ }^{7}$ The ANOVA showed a main effect of type of cuing $\left[F(2,118)=28.44, M S_{\mathrm{e}}=5.08, p<.0001, \eta^{2}=\right.$ .32] and a main effect of test $\left[F(1,118)=33.36, M S_{\mathrm{e}}=\right.$ $\left.0.91, p<.0001, \eta^{2}=.22\right]$, but no significant interaction $\left[F(2,118)=0.22, M S_{\mathrm{e}}=0.91, p=.80\right]$.

In particular, planned comparisons on the first test showed that the participants in the control group $(M=$ 5.27) generated a higher number of noncue options than did the participants in the rating group $(p<.0001 ; M=$ $3.25)$ and the reading group ( $p<.0001 ; M=2.75)$. The difference between the reading and the rating conditions was not significant. The participants produced slightly more options in the second test $(M=4.12)$ than in the first $(M=3.48)$. Planned comparisons on the second test showed exactly the same differences as those found on the first test: The participants in the control group $(M=$ $6.00)$ generated a higher number of noncue options than did the participants in the rating group $(p<.0001 ; M=$ $3.85)$ and the reading group ( $p<.0001 ; M=3.55)$. The difference between the reading and the rating conditions remained nonsignificant in the second test.

\section{Detailed Analysis of Generation Performance}

An in-depth analysis of Experiment 2A was carried out to separate the intracategorical effect of cues ("traditional" part-set cuing) from their intercategorical effects (partcategory cuing). Three sets of statistical analyses were performed on different dependent variables: (1) number of items generated (in cued/uncued response categories); (2) number of (cued/uncued) response categories accessed by participants; ${ }^{8}$ and (3) mean number of items generated per accessed category (see the Appendix for the enumeration of response categories). Due to space limitations, we will summarize here only the main findings. ${ }^{9}$

The participants in the control group generated significantly more noncue options in cued categories than did the participants in experimental conditions [intracategorical effect of cues: $F(2,118)=11.97, M S_{\mathrm{e}}=2.15, p<$ $.0001]$. Moreover, they also generated significantly more options in uncued categories than did the participants in the experimental groups [intercategorical effect of cues: $\left.F(2,118)=21.72, M S_{\mathrm{e}}=2.25, p<.0001\right]$. The analysis of response categories showed that the participants in the control group accessed a greater number of categories (both cued and uncued) than did the participants in the experimental groups $\left[F(2,118)=13.7, M S_{\mathrm{e}}=1.06, p<\right.$ .0001 , and $F(2,118)=18.33, M S_{\mathrm{e}}=1.31, p<.0001$, respectively]. Finally, we did not find significant differences in the mean number of options generated per accessed category (both cued and uncued) between the control group and the experimental groups.

\section{Discussion}

The results of the first generation test of Experiment $2 \mathrm{~A}$ fully supported the part-set cuing hypothesis. Moreover, the effect was robust to a variation in the cuing procedure: Reading or rating the cues yielded the same consequences. The persistence of the part-set cuing effect observed in the second generation test was predicted by the inhibition hypothesis, but not by the strategy disruption hypothesis. In fact, despite a slight general increase in the number of options generated from the first to the second test, no sign of attenuation of the part-set cuing effect was observed (see Figure 3).

Additional data analyses showed that the participants generated more options in the control group than in the experimental groups both for cued and for uncued categories. These findings can be explained by referring to the fact that the participants accessed more categories (both cued and uncued) in the control group (vs. the experimental groups). Therefore, cues seem to reduce option generation capacity both through an intracategorical effect ("traditional" part-set cuing that blocks generation from cued categories) and through an intercategorical effect (part-category cuing that blocks access to uncued categories). Both of these effects could be explained by inhibitory processes, given that they have always been observed also in the second generation test, when the cues were not presented.

\section{EXPERIMENT 2B}

The aim of Experiment 2B was to provide an explanation for the part-set cuing effect in option generation observed in the author scenario. In this experiment, we contrasted again the two explanations previously tested

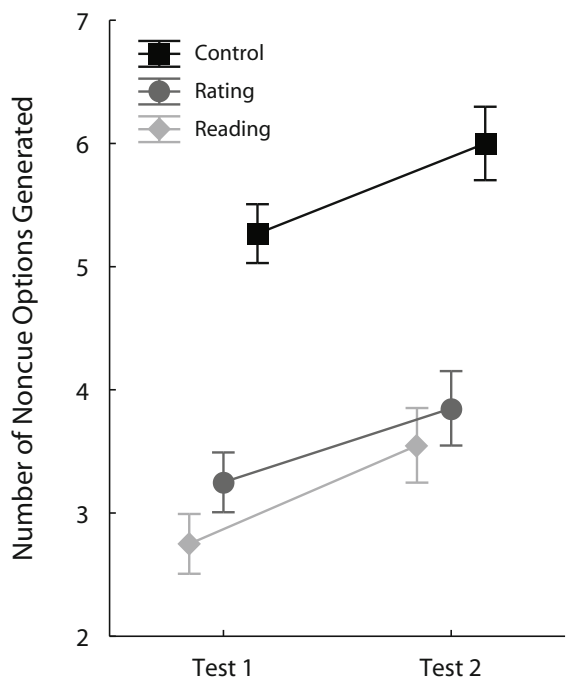

Figure 3. Number of noncue options generated in Experiment $2 \mathrm{~A}( \pm S E)$ as a function of experimental condition (control, rating, or reading) and of the generation task (Test 1 or Test 2). 
in Experiment 2A (inhibition and strategy disruption) by using the same test-retest paradigm.

\section{Method}

\section{Participants, Design, and Hypotheses}

Ninety-nine undergraduates recruited at the University of Trieste participated in Experiment 2B (20 of them male and 79 female; age, $M=20$ years, $S D=5.2$ ). Data were collected in class meetings. Experiment $2 \mathrm{~B}$ followed a 2 (cuing) $\times 2$ (test) mixed design, with random assignment of participants to the experimental group $(n=$ $49)$ or to the control group $(n=50)$. The experimental manipulation was analogous to the one used in the rating condition in Experiment 2A (i.e., the participants had to rate the cues).

We tested the same hypotheses as those put forward for Experiment 2A (part-set cuing; inhibition vs. strategy disruption). Even if generating preferred authors is a more common task than diagnosing radio problems, it did not appear to be a familiar task for our participants. According to a recent national-scale survey of reading habits (ISTAT, 2007) carried out on 24,000 households, the majority of Italians are weak readers $(31 \%$, one to three books per year) and light readers (25\%, four to six books per year). In line with the national survey, our participants were mainly weak readers $(25 \%)$ and light readers $(31 \%){ }^{10}$ These data suggest that generating authors was probably not a familiar task for our participants. Thus, we hypothesized that a highly effective generation strategy was not available to them, and we deemed it unlikely that the presentation of cues (names of authors) could harm such a generation strategy. ${ }^{11}$ Therefore, we considered it more likely also that the part-set cuing effects obtained in the author domain were due to the action of inhibitory processes. If the inhibition explanation is true, a comparable part-set cuing effect should be observed in the experimental group both in the first generation test (with the cues) and in the second generation test (without the cues). Thus, we expected to obtain a main effect of cuing but a nonsignificant cuing $\times$ test interaction.

\section{Procedure and Materials}

The wording of the author scenario was slightly changed in order to allow its presentation in isolation. However, as can be seen in Table 1, this did not affect in any way the information provided.

As in the previous experiments, the participants received a booklet, and they went through a series of steps timed by the experimenter. The participants in the experimental group had to read the author scenario and to evaluate for attractiveness of each of the 24 presented cues (as in the strong-cuing condition in Experiments 1A and $1 \mathrm{~B}$ ) in $3 \mathrm{~min}$. Then the scenario was presented again, together with the cues, and the participants had to generate the maximum possible number of authors in $5 \mathrm{~min}$. After the first generation task, the participants carried out a visuospatial filler task (find-thedifferences) for $3 \mathrm{~min}$. Finally, they were asked to perform a second generation task for $5 \mathrm{~min}$ (without the cues).

The participants in the control group started with a visuospatial filler task (find-the-differences), to be performed for $3 \mathrm{~min}$. Then they carried out the two generation tasks without the cues (for $5 \mathrm{~min}$ each). Between the two generation tasks, a second visuospatial filler task (find-the-differences) was performed for $3 \mathrm{~min}$. After the second generation task, all the participants were asked to fill out a brief questionnaire on their reading habits and to provide sociodemographic information.

\section{Results}

The results were analyzed with a 2 (cuing) $\times 2$ (test) ANOVA on the number of noncue options generated (Figure 4). The ANOVA showed a main effect of cuing $\left[F(1,97)=18.73, M S_{\mathrm{e}}=52.38, p<.0001, \eta^{2}=.16\right]$, whereas the effect of test $\left[F(1,97)=1.35, M S_{\mathrm{e}}=6.24\right.$,

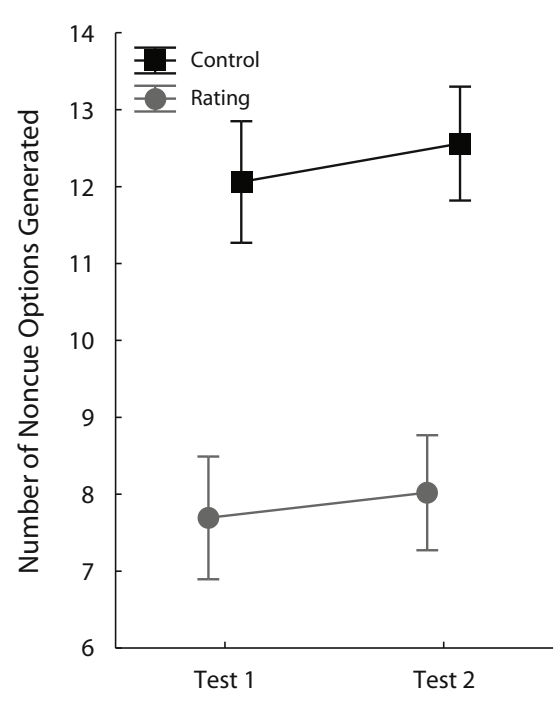

Figure 4. Number of noncue options generated in Experiment $2 \mathrm{~B}( \pm S E)$ as a function of experimental condition (control or rating) and of the generation task (Test 1 or Test 2$)$.

$p=.25]$ and the interaction $\left[F(1,97)=0.06, M S_{\mathrm{e}}=6.24\right.$, $p=.80]$ were not significant.

Planned comparisons on the first test showed that the participants in the control group $(M=12.06)$ generated a higher number of noncue options than did the participants in the cued (rating) group $(M=7.69, p<.001)$. The same significant difference was found also in the second test: The participants in the control group $(M=12.56)$ produced more options than did the participants in the cued group $(M=8.02, p<.0001)$.

A significant positive correlation was found between the number of noncue options generated and the number of books that the participants reported reading per year. This correlation was observed both in the cued group $(r=$ $.38, p<.01)$ and in the control group $(r=.47, p<.001)$. When the number of books read per year was introduced as a covariate in a 2 (cuing) $\times 2$ (test) ANCOVA on the number of noncue options generated, the pattern of results previously described did not change in any way.

\section{Discussion}

The results of Experiment 2B replicated the findings obtained in Experiment 2A, showing part-set cuing effects both in the first and in the second generation test. These findings provide further support for the inhibition explanation and they are not compatible with the strategy disruption account.

\section{GENERAL DISCUSSION}

The experiments described in this article were planned with two aims: (1) to show that part-set cuing effects in the generation of decision options can be reliably observed and (2) to provide an explanation for the part-set cuing 
effects in option generation. The results of four experiments highlighted clear part-set cuing effects. However, Experiments 1A and 1B showed that the effect may be attenuated by the type of decision scenario. A possibility is that the strength of the effect is related to how well established in memory the target category is and to the strength of preexperimental interitem associations (e.g., Bäuml \& Kuhbandner, 2003). Thus, an important goal for future research will be to define clear boundary conditions of part-set cuing in relation to participants' preexperimental knowledge and associations. Similarly, future research could investigate the boundary conditions of the effect in reference to the number of presented cues. Even if previous studies have shown that part-set cuing effects in option generation can be observed when a small number of cues are presented (cf. the Part-Set Cuing in Option Generation section), some findings of the present study suggest that the effects can be attenuated by a reduction in the number of cues.

Experiments 2A and 2B were planned so as to identify an explanation for the part-set cuing effects observed in Experiments 1A and 1B. To this aim, we contrasted the two accounts that are currently supported more by memory research (inhibition and strategy disruption) through the test-retest method. The results showed that the partset cuing effects we observed in our option generation scenarios are lasting, thus indicating that inhibition is the most likely explanation of our findings. ${ }^{12}$ Detailed analyses carried out on the results of Experiment 2A showed that the presentation of cues reduces option generation capacity from both cued categories (traditional part-set cuing effect) and uncued categories (part-category cuing). It is likely that both these effects are related to inhibitory processes, given that they have been observed in both the first and the second generation tasks.

The results of the present study bear implications for option generation and memory research. We showed that part-set cuing can take place in the generation of decision options, that it is resistant to variations in the cuing procedure, and that it is probably caused by inhibitory processes in the domains investigated. ${ }^{13}$ These findings significantly advance our knowledge of part-set cuing in option generation. However, an important avenue for future research would be to examine to what extent the effect of part-set cuing on option generation affects judgment and choice, given that the few existing studies do not provide conclusive answers. In the long term, this research could complement memory-based decision theories (e.g., Stewart et al., 2006; Thomas et al., 2008) with a specification of the processes underlying part-set cuing and its effects on decision behavior.

For what concerns memory research, our results support the generality of part-set cuing, and they are compatible with the inhibition explanation. Moreover, we showed that the effect can be observed also in older participants' generation performance, underlining the need to provide a better characterization of part-set cuing in aging. Although the generalizability of memory laws and empirical regularities is currently being debated (Roediger, 2008), this article offers a contribution in the opposite direction.

\section{AUTHOR NOTE}

Portions of this work were presented in poster format at SJDM 2007 (the 28th annual meeting of the Society for Judgment and Decision Making). The authors thank two anonymous reviewers, Mike Oaksford, and Francesco Marcatto for their detailed comments. Correspondence concerning this article should be addressed to F. Del Missier, Department of Psychology, University of Trieste, via S. Anastasio 12, I-34134, Trieste, Italy (e-mail: delmisfa@units.it).

\section{REFERENCES}

Alba, J. W., \& Chattopadhyay, A. (1985). Effects of contest and partcategory cues on recall of competing brands. Journal of Marketing Research, 22, 340-349.

Anderson, M. C., BJork, E. L., \& BJork, R. A. (1994). Remembering can cause forgetting: Retrieval dynamics in long-term memory. Journal of Experimental Psychology: Learning, Memory, \& Cognition, 20, 1063-1087.

Anderson, M. C., \& BJork, R. A. (1994). Mechanisms of inhibition in long-term memory: A new taxonomy. In D. Dagenbach \& T. Carr (Eds.), Inhibitory processes in attention, memory, and language (pp. 265-326). New York: Academic Press.

Aslan, A., BäUmL, K.-H., \& Grundgeiger, T. (2007). The role of inhibitory processes in partlist cuing. Journal of Experimental Psychology: Learning, Memory, \& Cognition, 33, 335-341.

Barsalou, L. W. (1983). Ad hoc categories. Memory \& Cognition, 11, 211-227.

Basden, B. H., Basden, D. R., \& Stephens, J. P. (2002). Part-set cuing of order information in recall tests. Journal of Memory \& Language, 47, 517-529.

BASDEN, D. R., \& BASDEN, B. H. (1995). Some tests of the strategy disruption hypothesis of part-list cuing inhibition. Journal of Experimental Psychology: Learning, Memory, \& Cognition, 21, 1656-1669.

Basden, D. R., Basden, B. H., \& Galloway, B. C. (1977). Inhibition with part-list cuing: Some tests of the item strength hypothesis. Journal of Experimental Psychology: Human Learning \& Memory, 3, 100-108.

BÄUmL, K. H., \& Aslan, A. (2004). Part-list cuing as instructed retrieval inhibition. Memory \& Cognition, 32, 610-617.

BÄUML, K. H., \& ASLAN, A. (2006). Part-list cuing can be transient and lasting: The role of encoding. Journal of Experimental Psychology: Learning, Memory, \& Cognition, 32, 33-43.

BÄUmL, K.-H., \& KuhbandneR, C. (2003). Retrieval-induced forgetting and part-list cuing in associatively structured lists. Memory \& Cognition, 31, 1188-1197.

Brown, J. (1968). Reciprocal facilitation and impairment of free recall. Psychonomic Science, 10, 41-42.

Del Missier, F., Ferrante, D., \& Costantini, E. (2007). Focusing effects in predecisional information acquisition. Acta Psychologica, 125, 155-174.

EDGington, E. S. (1972). A normal curve method for combining probability values from independent experiments. Journal of Psychology, 82, 85-89.

FISHER, R. A. (1948). Combining independent tests of significance. American Statistician, 2, 30.

Foos, P. W., \& Clark, M. C. (2000). Old age, inhibition, and the part-set cuing effect. Educational Gerontology, 26, 155-160.

Hudson, R. L., \& Austin, J. B. (1970). Effect of context and category name on the recall of categorized word lists. Journal of Experimental Psychology, 86, 43-47.

Hultsch, D. F., \& Craig, E. R. (1976). Adult age differences in the inhibition of recall as a function of retrieval cues. Developmental Psychology, 12, 83-84.

ISTAT (2007). La lettura di libri in Italia [Book-reading habits in Italy]. Retrieved from www.istat.it/salastampa/comunicati/ non_calendario/20070510_00/.

Johnson, J., \& RAAB, M. (2003). Take the first: Option generation and resulting choices. Organizational Behavior \& Human Decision Processes, 91, 215-229.

KARCHMER, M. A., \& WinOGRAD, E. (1971). Effects of studying a subset of familiar items on recall of the remaining items: The John Brown effect. Psychonomic Science, 25, 224-225. 
KISSLER, J., \& B̈̈UML, K. H. (2005). Memory retrieval in schizophrenia: Evidence from part-list cuing. Journal of the International Neuropsychological Society, 11, 273-280.

Marsh, E. J., Dolan, P. O., Balota, D. A., \& Roediger, H. L., III (2004). Part-set cuing effects in younger and older adults. Psychology \& Aging, 19, 134-144.

Mehle, T. (1982). Hypothesis generation in an automobile malfunction inference task. Acta Psychologica, 52, 87-106.

Moulin, C. (2000, April). When it is good to forget: Evidence for intact retrieval inhibition in older adults. Poster presented at the meeting of the Cognitive Aging Society, Atlanta.

NICKERSON, R. S. (1984). Retrieval inhibition from part-set cuing: A persisting enigma in memory research. Memory \& Cognition, 12, 531-552.

Oswald, K. M., Serra, M., \& Krishna, A. (2006). Part-list cuing in speeded recognition and free recall. Memory \& Cognition, 34, 518526.

Pei, B. K. W., \& Tuttle, B. M. (1999). Part-set cuing effects in a diagnostic setting with professional auditors. Journal of Behavioral Decision Making, 12, 233-256.

PeYnirCIOUĞLU, Z. F. (1987). On the generality of the part-set cuing effect: Evidence from nonmemory tasks. Journal of Experimental Psychology: Learning, Memory, \& Cognition, 13, 437-442.

RoEDIGER, H. L., III (2008). Relativity of remembering: Why the laws of memory vanished. Annual Review of Psychology, 59, 225-254.

Roediger, H. L., III, \& NeEly, J. H. (1982). Retrieval blocks in episodic and semantic memory. Canadian Journal of Psychology, 36, 213-242.

Roediger, H. L., III, Stellon, C. C., \& Tulving, E. (1977). Inhibition from part-list cues and rate of recall. Journal of Experimental Psychology: Human Learning \& Memory, 3, 174-188.

Roediger, H. L., III, \& Tulving, E. (1974, May). Part-list cueing and directing forgetting. Paper presented at the meeting of the Midwestern Psychological Association, Chicago.

RunDus, D. (1973). Negative effects of using list items as recall cues. Journal of Verbal Learning \& Verbal Behavior, 12, 43-50.

Serra, M., \& Oswald, K. M. (2006). Part-list cuing of associative chains: Tests of strategy disruption. Journal of General Psychology, 133, 301-317.

Slamecka, N. J. (1968). An examination of trace storage in free recall. Journal of Experimental Psychology, 76, 504-513.

SlamecKa, N. J. (1969). Testing for associative storage in multitrial free recall. Journal of Experimental Psychology, 81, 557-560.

Sloman, S. A. (1991). Part-set cuing inhibition in category-instance and reason generation. Bulletin of the Psychonomic Society, 29, 136-138.

Sloman, S. [A.], Rottenstreich, Y., Wisniewski, E., HadjichrisTIDIs, C., \& Fox, C. R. (2004). Typical versus atypical unpacking and superadditive probability judgment. Journal of Experimental Psychology: Learning, Memory, \& Cognition, 30, 573-582.

Stewart, N., Chater, N., \& Brown, G. D. A. (2006). Decision by sampling. Cognitive Psychology, 53, 1-26.

Thomas, R. P., Dougherty, M. R., Sprenger, A., \& Harbison, J. I. (2008). Diagnostic hypothesis generation and human judgment. Psychological Review, 115, 155-185.

Tombinson, T. D. (2007). The role of part set cuing and retrievalinduced forgetting in subjective probability judgments. Unpublished master's thesis, University of Maryland. Retrieved from hdl.handle net/1903/7266.

\section{NOTES}

1. The editing explanation assumes that participants in the cuing condition need to check whether each item they have just retrieved (or generated) is valid (i.e., it is not a member of the cue set) before writing the response. This check requires time, and thus participants in the cuing condition may be able to perform fewer retrieval attempts than can participants in the control condition.

2. In Experiment 1A, half of the participants in the weak- and strongcuing conditions received the cues in random order, and the other half in a category-blocked order. Given that the results did not show any difference related to the order of presentation of the cues, data were pooled. Cues were always presented in category-blocked order in the other experiments reported in this article.

3. The deadline of $5 \mathrm{~min}$ for each scenario was established after a pilot study showed that the great majority of participants were unable to generate additional options after that time.

4. This rating task was devised in order to be sure that the participants read the cues attentively. We regard this manipulation as more natural and less intrusive for a decision task than asking participants to study the cues for minutes (as usually happens in part-set cuing studies). However, Experiment 2A employed an even less obtrusive manipulation (i.e., just reading the cues)

5. Data analysis was performed both on untransformed data and after a square-root transformation. Given that we always obtained converging results, we present analyses and graphs based on untransformed data, in order to increase their readability. We removed participants who generated a number of options 1.5 interquartile ranges below the first quartile or above the third quartile of their respective condition (two cases in Experiment 1A and eight cases in Experiment 1B).

6. A similar consideration was advanced by Marsh et al. (2004, p. 135) in their discussion of Hultsch and Craig's (1976) results.

7. In the second test, we considered the following as noncue options: (1) noncue items generated for the first time in the second test and (2) noncue items generated in both the first and the second tests.

8. A category is accessed if the participant generates at least one noncue item from that category. A cued category is a category containing items used as cues in the experiment, whereas an uncued category is a category whose items were not used as cues. Response categories and cues are presented in the Appendix. Responses to Experiment 2A were classified by two independent judges, with an interrater agreement of $95 \%$. Disagreements were resolved after a joint discussion.

9. A detailed report of the results is available from the first author.

10. These percentages could have been underestimated due to the influence of social desirability concerns, because the participants' answers were collected in an academic setting.

11. This does not mean that our participants did not use any strategy in the author generation task (such as a genre-based strategy). It means that this strategy was probably constructed on the spot and was not very effective.

12. Our results do not exclude the possibility that strategy disruption may explain part-set cuing effects in decision domains involving the adoption of well-practiced and effective generation strategies. Assuming that part-set cuing effects could be observed in these domains, this possibility is worth investigating in future studies. An alternative explanation of our findings might involve a process of retrieval competition (Rundus, 1973). However, several studies have challenged the general validity of the retrieval competition account (see Bäuml \& Aslan, 2006)

13. This conclusion, although based on the absence of a significant interaction, is convincingly supported by the data. First, the absence of a significant interaction was consistently observed in three tests carried out in two different experiments. The combined probability of the interaction effect in these tests, computed with Fisher's (1948) method, was nonsignificant and very close to $1\left[\chi^{2}(6)=0.736, p=.997\right]$. Similar results were obtained with Edgington's (1972) method. Moreover, the predicted absence of an interaction effect in Experiments 2A and 2B always implies finding significant part-set cuing effects in generation tests. These significant differences were always observed. Finally, as can be seen in Figures 3 and 4, there is no sign of attenuation of the part-set cuing effect in the repeated tests of Experiments $2 \mathrm{~A}$ and $2 \mathrm{~B}$. 


\section{APPENDIX}

Response Categories and Cues for Each Scenario

Cued and uncued response categories are presented. Each cued category is followed by the cues used in the experiments. In Experiments 1A and 1B, items marked with (SC) were used only in the strong-cuing condition in Experiments $1 \mathrm{~A}$ and $1 \mathrm{~B}$. All other cues were used both in the strong-cuing condition and in the weak-cuing condition in the same experiments. In the rating and reading conditions in Experiment $2 \mathrm{~A}$ and in the cued condition in Experiment $2 \mathrm{~B}$ (rating), we used the same cues as those in the strong-cuing conditions in Experiments $1 \mathrm{~A}$ and $1 \mathrm{~B}$.

\section{Radio Scenario (Experiments 1A, 1B, 2A)}

1. Radio technical problem.

2. External intervention on the radio: someone disconnected the radio plug; someone moved the antenna; a pet pushed down the radio; someone lowered the volume.

3. Problem related to the speaker: speaker's technical error (SC); speaker's silence (SC).

4. Problem related to the program: breaking news; a minute of silence; advertising; strike.

5. Technical problem on the source side: technical problem in the studio; technical problem with the microphone; technical interruption of the program; interruption of a journalist's report.

6. Problem related to the signal.

7. Problem related to the external environment: A door was closed (SC); a noise overshadowed the voice (SC).

8. Problem related to electrical power.

9. Problem related to the listener.

10. Other.

\section{Gift Scenario (Experiments 1A, 1B)}

1. Clothing and accessories: scarf; hat; stockings/socks; sweatshirt; belt; wallet.

2. Objects that can be used for recreation/leisure time: ticket for the cinema; subscription to a magazine; ticket for an exhibition; music CD; film DVD; ticket for a concert.

3. Communication and technology.

4. Stationery and fine arts: pencils; drawing set; pocket diary; picture/paint; pen case; exercise books.

5. Personal care.

6. Food items: bottle of wine (SC); chocolates (SC); coffe box (SC); a dinner in a pizzeria (SC).

7. Decoration/house items: photo frame (SC), flowers (SC).

8. Other.

\section{Author Scenario (Experiments 1A, 1B, 2B)}

1. Italian authors of novels: Calvino; Svevo; Sciascia; Verga; Tamaro; Pirandello.

2. Non-Italian authors of novels: Coelho (SC); Poe (SC); Saramago (SC); Nabokov (SC); Auster (SC); Blixen (SC).

3. Authors of crime fiction: Christie; Simenon; Cornwell; Camilleri; Faletti; Conan Doyle.

4. Authors of political or historical essays: Fallaci; Bobbio; Pansa; Veronesi; Le Goff; De Felice.

5. Classical authors (Greeks and Romans).

6. Authors of fantasy and science fiction.

7. Authors of children's books.

8. Authors of poetry and drama.

9. Other. 